\title{
ESTUDO DO EFEITO DO CLORETO EM CATALISADORES DE PALÁDIO USANDO A ANÁLISE NO INFRAVERMELHO E DE FOTOELÉTRONS EXCITADOS POR RAIOS X
}

\author{
Eduardo V. Cunha, Flávio Faccin, Celso C. Moro e Edilson V. Benvenutti* \\ Instituto de Química, Universidade Federal do Rio Grande do Sul, CP 15003, 91501-970 Porto Alegre - RS \\ Sandra C. de Castro ${ }^{\dagger}$ \\ Instituto de Física Gleb Wataghin, Universidade Estadual de Campinas, CP 6165, 13083-970 Campinas - SP
}

Recebido em 24/4/01; aceito em 4/9/01

\begin{abstract}
INFRARED AND X-RAY PHOTOELECTRON SPECTROSCOPY STUDY OF CHLORIDE EFFECT IN PALLADIUM CATALYSTS. Chloride poisoning is known as having an inhibitor effect in the activity of metal catalysis. In this work in situ infrared spectroscopy (FTIR) of adsorbed carbon monoxide and x-ray photoelectron spectroscopy (XPS) were used to investigate the effect of chloride presence in the electronic metal density in the $\mathrm{d}$ subshell of palladium dispersed on alumina. The chloride poisoning effect was interpreted as an electronic effect since a weak back-bonded Pd-CO was formed due to the decrease in the electronic density of the $\mathrm{d}$ subshell of palladium, which could be also detected by the higher $\mathrm{Pd} 3 \mathrm{~d}_{5 / 2}$ binding energy in the chloride presence. A similar poisoning effect was also observed for chloride free $\mathrm{Pd} / \mathrm{Al}_{2} \mathrm{O}_{3}$ reduced at $500{ }^{\circ} \mathrm{C}$, and it was interpreted based on the interaction of metal with the alumina surface. The use of molybdena/alumina binary system as support, yield a contrary effect due to the metal-support interaction.
\end{abstract}

Keywords: chloride poisoning effect; infrared; XPS.

\section{INTRODUÇÃO}

As propriedades catalíticas de catalisadores metálicos suportados são geralmente influenciadas pela presença de aditivos, que podem ser tanto promotores ${ }^{1,2}$ como também inibidores ${ }^{3-6}$. Alguns inibidores como cloreto, as vezes, podem estar presente no catalisador, não como aditivos, mas sim, como resíduo dos sais metálicos precursores do catalisador, e podem produzir a diminuição na atividade catalítica $^{3,7,8}$.

A influência do cloreto na atividade catalítica pode ser interpretada de duas formas: i) Como um efeito estérico, onde o haleto coordena-se aos sítios que são necessários a adsorção e ativação de outras espécies ${ }^{9-11}$. Nesse caso o cloreto produz o bloqueio dos sítios metálicos resultando na diminuição da acessibilidade dos reagentes a esses sítios ${ }^{11,12}$. ii) A outra interpretação é considerar o envenenamento do cloreto como um efeito eletrônico. Nesse caso cloreto metálico pode ser formado ${ }^{13,14}$. Para metais nobres como paládio, esses haletos são mais estáveis que os óxidos ${ }^{13,15}$. A atividade catalítica portanto diminui, pois os haletos metálicos são menos ativos que as partículas metálicas.

Nesse trabalho, nossos resultados evidenciam que a presença de cloreto no catalisador metálico produz efeitos eletrônicos e que quando esse é reduzido a temperatura de $500{ }^{\circ} \mathrm{C}$, a interação do paládio disperso com o oxigênio do suporte alumina pode também resultar em um efeito semelhante ao do cloreto. Uma redução parcial do efeito do envenenamento por cloreto pode ser obtida quando usa-se molibdênio/alumina como suporte. As interpretações foram feitas a partir da análise através da espectroscopia de fotoelétrons excitados por raios-X e também na análise das ligações por retro-doação $p i$ entre o metal e a carbonila, que dependem da densidade eletrônica

*e-mail: edilson@iq.ufrgs.br

†in memoriam do metal. Essas ligações podem ser monitoradas a partir da espectroscopia no infravermelho.

\section{PARTE EXPERIMENTAL}

\section{Síntese do $\mathrm{Pd} / \mathrm{Al}_{2} \mathrm{O}_{3}$ (sem cloreto)}

O suporte $\gamma$-alumina $\left(150 \mathrm{~m}^{2} \mathrm{~g}^{-1} \mathrm{BET}\right)$ foi ativado sob vácuo a $120{ }^{\circ} \mathrm{C}$, por $3 \mathrm{~h}$. Acetilacetonato de paládio (Aldrich) (107 mg) foi dissolvido em $10 \mathrm{~mL}$ de acetona, sob aquecimento. Essa solução foi pingada sob agitação constante sobre $3,7 \mathrm{~g}$ de alumina ativada. $\mathrm{O}$ sólido resultante foi seco por $5 \mathrm{~h}$ a $110{ }^{\circ} \mathrm{C}$ e calcinado por $4 \mathrm{~h}$ a $450{ }^{\circ} \mathrm{C}$. A massa do precursor metálico foi calculada visando obter o catalisador com $1 \%$ em massa de paládio.

\section{Síntese do $\mathrm{Pd} / \mathrm{Al}_{2} \mathrm{O}_{3}$ (com cloreto)}

$\mathrm{O} \mathrm{PdCl}_{2}$ (Merck) (51mg) foi dissolvido em 1,5 mL de $\mathrm{HCl}$ $\left(6 \mathrm{~mol} \mathrm{~L}^{-1}\right)$ sob aquecimento. Essa solução foi pingada sobre $3 \mathrm{~g} \mathrm{de}$ $\gamma$-alumina, sob agitação. O sólido resultante foi seco por $5 \mathrm{~h}$ a $110^{\circ} \mathrm{C}$ e calcinado por $4 \mathrm{~h}$ a $450{ }^{\circ} \mathrm{C}$. A massa do precursor metálico foi calculada visando obter $1 \%$ em massa de paládio no catalisador.

\section{Síntese do $\mathrm{Pd} / \mathrm{MoO}_{3} / \mathrm{Al}_{2} \mathrm{O}_{3}$ (sem cloreto)}

Isopropóxi de alumínio e acetilacetonato de paládio foram dissolvidos em álcool isopropílico. O processo de hidrólise foi feito pela adição da mistura água e álcool isopropílico. O gel resultante foi seco a $110^{\circ} \mathrm{C}$ por $18 \mathrm{~h} \mathrm{e} \mathrm{calcinado} \mathrm{a} 450^{\circ} \mathrm{C}$ por $5 \mathrm{~h}$. Acetilacetonato de paládio (Aldrich) $(107 \mathrm{mg}$ ) foi dissolvido em $10 \mathrm{~mL}$ de acetona, sob aquecimento. Essa solução foi pingada sobre o suporte $\mathrm{MoO}_{3} /$ $\mathrm{Al}_{2} \mathrm{O}_{3}(3,7 \mathrm{~g})$, sob agitação constante. $\mathrm{O}$ sólido resultante foi seco por $5 \mathrm{~h}$ a $110{ }^{\circ} \mathrm{C}$ e calcinado por $4 \mathrm{~h}$ a $450{ }^{\circ} \mathrm{C}$. A massa do precursor metálico foi calculada visando obter o catalisador com $1 \%$ em massa de paládio. 


\section{Tratamento Térmico Redutivo}

Foram preparados discos dos catalisadores com $5 \mathrm{~cm}^{2}$ e $c a$. $60 \mathrm{mg}$ de massa. Os discos foram inicialmente aquecidos a $300{ }^{\circ} \mathrm{C}$ sob vácuo $\left(10^{-5}\right.$ Torr $)(1$ Torr $=133.3 \mathrm{~Pa})$ durante 1 hora e expostos a 300 Torr de oxigênio por $1 \mathrm{~h}$, a $300^{\circ} \mathrm{C}$. O oxigênio foi degasado durante $1 \mathrm{~h}$, na mesma temperatura, e então os discos foram submetidos a 200 Torr de hidrogênio, na temperatura de $200{ }^{\circ} \mathrm{C}$ (LTR Low Temperature of Reduction) ou $500{ }^{\circ} \mathrm{C}(\mathrm{HTR}$ - High Temperature of Reduction), por $1 \mathrm{~h}$. Os catalisadores foram finalmente degasados a $300^{\circ} \mathrm{C}$ por $1 \mathrm{~h}$, sob vácuo.

\section{Análise no Infravermelho (FTIR)}

Os discos de catalisadores foram analisados in situ na região do infravermelho após terem sido submetidos ao tratamento redutivo, usando-se um espectrofotômetro Bomem FT-IR, modelo MB-102. Uma cela de quartzo ${ }^{16}$ foi conectada a uma linha de vácuo livre de graxa através da qual foi possível submeter os catalisadores ao monóxido de carbono, sob pressão controlada. Os espectros foram obtidos com uma resolução de $2 \mathrm{~cm}^{-1}$, com 150 varreduras.

\section{Análise por Espectroscopia de Fotoelétrons Excitados por Raios X (XPS)}

As medidas de XPS foram feitas usando-se um equipamento McPherson ESCA-36 com radiação fonte $\mathrm{Al} \mathrm{K} \alpha(1486,6 \mathrm{eV})$ e pressão base de $10^{-7}$ Torr. A calibração das energias de ligação foram feitas usando-se $\mathrm{C} 1 \mathrm{~s}$ do carbono presente nas amostras como contaminante $(284,6 \mathrm{eV})$. As razões atômicos foram calculadas integrando-se os picos das áreas corrigidas pelo método de fotoionização de Scofield ${ }^{17}$.

\section{RESULTADOS}

\section{Análise no Infravermelho (FTIR)}

Os espectros de $\mathrm{Pd} / \mathrm{Al}_{2} \mathrm{O}_{3}$ sem cloreto expostos ao monóxido de carbono são mostrados na Figura 1. Na Figura 1a, o espectro foi obtido para amostra submetida ao tratamento LTR. A banda em 2080 $\mathrm{cm}^{-1}$ foi atribuída a estiramento de carbonila linearmente coordenada no paládio disperso enquanto que a banda em $1938 \mathrm{~cm}^{-1}$ foi atribuída a espécies $\mu$-CO. Para as amostras submetidas ao tratamento HTR (Figura 1b) as bandas devidas a estiramento da carbonila coordenada na forma linear e também em ponte sofrem um deslocamento para valores mais energéticos, 2086 e $1963 \mathrm{~cm}^{-1}$, respectivamente.

Os espectros do $\mathrm{Pd} / \mathrm{Al}_{2} \mathrm{O}_{3}$ com cloreto, tanto submetido ao tratamento LTR como também ao HTR, são mostrados na Figura 2. Após tratamento LTR, observou-se que a banda devida a carbonila linear deslocou-se para maior valor de número de onda $\left(2102 \mathrm{~cm}^{-1}\right)$ (maior energia) quando comparada com os espectros obtidos para o catalisador $\mathrm{Pd} / \mathrm{Al}_{2} \mathrm{O}_{3}$ sem cloreto $\left(2080 \mathrm{~cm}^{-1}\right)$. No caso da amostra submetida ao tratamento HTR, o deslocamento pode também ser observado para as bandas de espécies $\mu$-CO (Figura $2 \mathrm{~b}$ ). Na presença de cloreto, após tratamento HTR, as posições das bandas de estiramento da carbonila são 2102 e $1979 \mathrm{~cm}^{-1}$ para linear e $\mu$-CO, respectivamente.

Os espectros do $\mathrm{Pd} / \mathrm{MoO}_{3} / \mathrm{Al}_{2} \mathrm{O}_{3}$ sem cloreto são mostrados na Figura 3. As bandas de carbonila linear aparecem em 2109 e $2096 \mathrm{~cm}^{-1}$ para amostras submetidas aos tratamentos LTR e HTR, respectivamente.

A pressão de monóxido de carbono usada nesse trabalho (10 Torr) foi suficiente para produzir a saturação dos sítios de paládio, visto que não foi observado aumento nas áreas sob as bandas para maiores pressões.

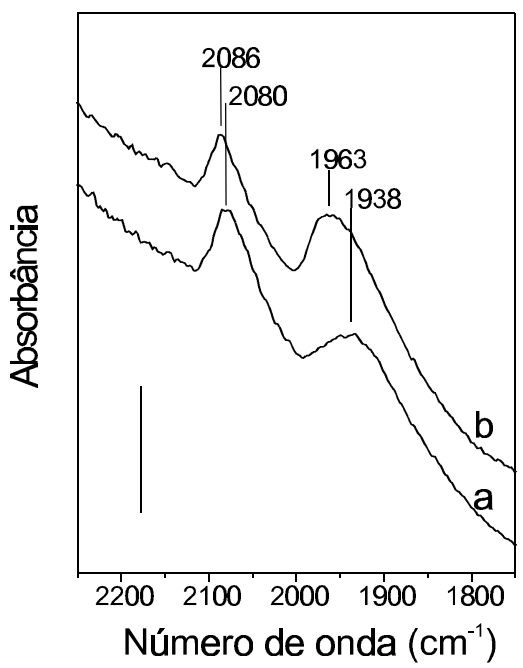

Figura 1. Espectros no infravermelho do catalisador $\mathrm{Pd} / \mathrm{Al}_{2} \mathrm{O}_{3}$ sem cloreto, exposto a 10 Torr de monóxido de carbono: a) Submetido a tratamento LTR; b) Submetido a tratamento HTR. O valor da barra é 0,2

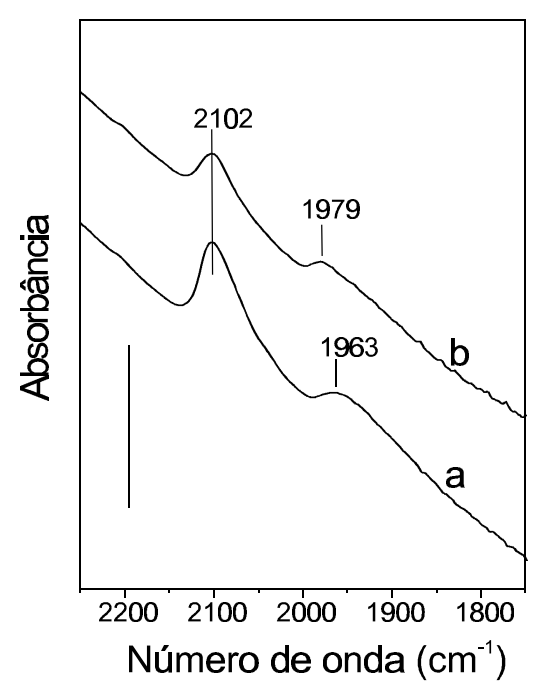

Figura 2. Espectros no infravermelho do catalisador $\mathrm{Pd} / \mathrm{Al}_{2} \mathrm{O}_{3}$ com cloreto, exposto a 10 Torr de monóxido de carbono: a) Submetido a tratamento LTR; b) Submetido a tratamento HTR. O valor da barra é 0,2

\section{Análise por Espectroscopia de Fotoelétrons Excitados por Raios X (XPS)}

Os espectros de XPS obtidos para amostras de $\mathrm{Pd} / \mathrm{Al}_{2} \mathrm{O}_{3}$ com cloreto e $\mathrm{Pd} / \mathrm{Al}_{2} \mathrm{O}_{3}$ sem cloreto submetidas ao tratamento LTR e para amostra de $\mathrm{Pd} / \mathrm{MoO}_{3} / \mathrm{Al}_{2} \mathrm{O}_{3}$ submetida ao tratamento HTR são mostrados na Figura 4. Pode-se observar um deslocamento da energia de ligação do $\mathrm{Pd} 3 \mathrm{~d}_{5 / 2}$ para valores mais baixos (espectros a para c). $\mathrm{O}$ valor mais alto de energia de ligação do $\mathrm{Pd} 3 \mathrm{~d}_{5 / 2}$ foi obtido para amostra de $\mathrm{Pd} / \mathrm{Al}_{2} \mathrm{O}_{3}$ com cloreto, enquanto que o valor mais baixo foi o do $\mathrm{Pd} / \mathrm{MoO}_{3} / \mathrm{Al}_{2} \mathrm{O}_{3}$ sem cloreto (Tabela 1 ).

\section{DISCUSSÃO}

Quando o paládio encontra-se reduzido, a densidade eletrônica no subnível d pode produzir uma eficiente ligação pi (retro-doação) entre o paládio e a carbonila, assim a ligação C-O fica enfraquecida. Portanto, no espectro infravermelho, a energia da banda da carbonila 


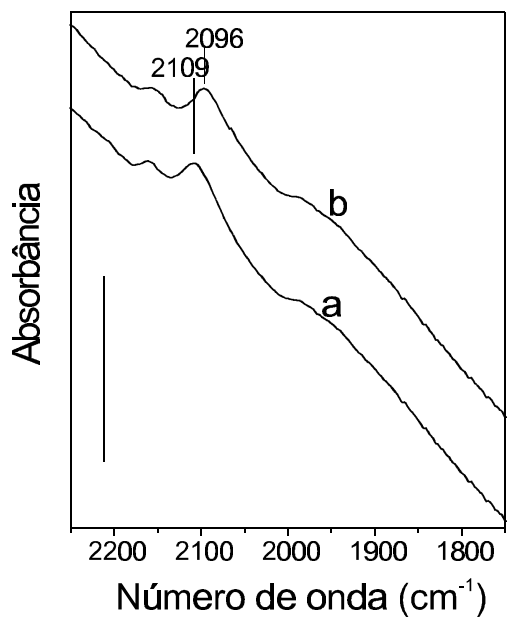

Figura 3. Espectros no infravermelho do catalisador $\mathrm{Pd} / \mathrm{MoO}_{3} / \mathrm{Al}_{2} \mathrm{O}_{3}$ exposto a 10 Torr de monóxido de carbono. a) Submetido a tratamento LTR; b) Submetido a tratamento HTR. O valor da barra é 0,3

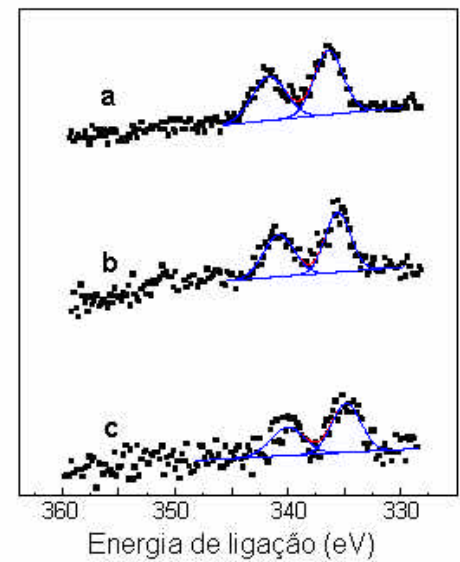

Figura 4. Espectros de XPS mostrando a banda $P d 3 d_{5 / 2}$ do paládio nos catalisadores: a) $\mathrm{Pd}_{2} \mathrm{Al}_{2} \mathrm{O}_{3}$ com cloreto; b) $\mathrm{Pd}_{2} \mathrm{Al}_{2} \mathrm{O}_{3}$ sem cloreto; c) $\mathrm{Pd} /$ $\mathrm{MoO}_{3} / \mathrm{Al}_{2} \mathrm{O}_{3}$

coordenada ao paládio está inversamente relacionada à densidade eletrônica do metal.

No espectro do $\mathrm{Pd} / \mathrm{Al}_{2} \mathrm{O}_{3}$ sem cloreto, submetido ao tratamento LTR (Figura 1a), as posições das bandas de carbonila linear e em ponte em 2080 e $1938 \mathrm{~cm}^{-1}$, respectivamente, correspondem a paládio disperso sobre alumina, na forma reduzida, na ausência de efeito produzido por envenenamento por cloreto. Seria esperado que após tratamento a $500{ }^{\circ} \mathrm{C}$ o paládio sofresse uma redução ainda mais eficiente, contudo no espectro $1 \mathrm{~b}$, obtido após a amostra ter sido submetida a tratamento HTR, as bandas sofrem um deslocamento para maiores valores de número de onda quando comparadas ao espectro 1a. Isso é uma indicação de que a densidade eletrônica no subnível d do metal sofreu uma redução, e pode somente ser interpretada considerando-se a interação do paládio disperso com os oxigênios da alumina. $\mathrm{Al}_{2} \mathrm{O}_{3}$ é um típico suporte não redutível, portanto o metal disperso está diretamente ligado aos oxigênios da superfície. $\mathrm{O}$ tratamento HTR produz portanto uma forte interação metal-oxigênio. Essa interação já foi sugerida por outros, para o catalisador $\mathrm{Pd} /$ $\mathrm{Al}_{2} \mathrm{O}_{3}^{3,8,18,19}$ e é também evidenciada pelos resultados obtidos na análise de XPS, que será discutida abaixo.
Para amostra de $\mathrm{Pd} / \mathrm{Al}_{2} \mathrm{O}_{3}$ com cloreto, exposto a monóxido de carbono, a posição das bandas de estiramento da carbonila surgem em números de onda maiores (Figura 2) quando comparada aos espectros de amostras sem cloreto (Figura 1). Esse comportamento é também devido ao decréscimo na densidade eletrônica do subnível d do metal disperso, entretanto nesse caso, produzido pelo cloreto. $\mathrm{Na}$ presença de cloreto, o paládio não pode ser completamente reduzido, devido a formação de espécies $\mathrm{Pd}-\mathrm{Cl}^{13,15}$.

Portanto, a presença de cloreto no catalisador $\mathrm{Pd} / \mathrm{Al}_{2} \mathrm{O}_{3}$, tanto em amostra submetida a tratamento LTR como também HTR, ou ainda a interação metal-oxigênio em amostras de $\mathrm{Pd} / \mathrm{Al}_{2} \mathrm{O}_{3}$ sem cloreto, quando submetida a tramento HTR, produzem um decréscimo na densidade eletrônica do paládio.

Por outro lado, se suportes com propriedades redutíveis como $\mathrm{TiO}_{2}$ ou $\mathrm{MoO}_{3}$ são usados, o tratamento HTR pode produzir o efeito SMSI (Strong Meta-Support Interactions) entre o paládio disperso e

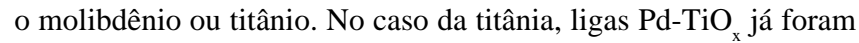
observadas $^{20-23}$. O efeito SMSI aumenta a densidade eletrônica do subnível d do metal disperso ${ }^{23-26}$, assim uma forte ligação pi (retrodoação) entre o paládio e a carbonila pode ser formada. No espectro da Figura 3b, obtido para o catalisador $\mathrm{Pd} / \mathrm{MoO}_{3} / \mathrm{Al}_{2} \mathrm{O}_{3}$ após tratamento HTR, um deslocamento da banda da carbonila para menores valores de número de onda pode ser observado, quando comparado com o da Figura 3a, obtido para a amostra submetida ao tratamento LTR.

O tratamento HTR $\left(500{ }^{\circ} \mathrm{C}\right)$ na alumina produz um decréscimo na densidade eletrônica do paládio disperso devido a forte interação com os oxigênios da superfície. Esse efeito é similar ao efeito eletrônico produzido pelo envenenamento por cloreto. No caso do suporte redutível molibdênia/alumina, o efeito SMSI, evidente após tratamento HTR, resulta em um efeito inverso, onde o paládio fica fortemente ligado à carbonila por retro-doação pi. Nessa situação o oxigênio da carbonila torna-se mais suscetível para reagir em processos catalíticos dissociativos. Já foi relatado que os metais quando dispersos em suportes redutíveis, tratados sob HTR, produzem um aumento da seletividade em reações de hidrogenação de $\mathrm{C}=\mathrm{O}$ com relação a $\mathrm{C}=\mathrm{C}^{27-29}$.

A energia de ligação Pd $3 \mathrm{~d}_{5 / 2}$, obtida na análise de XPS, é mostrada na Tabela 1 e está em concordância com os resultados da análise no infravermelho. Para a amostra de $\mathrm{Pd} / \mathrm{Al}_{2} \mathrm{O}_{3}$ sem cloreto, submetida a tratamento LTR, a energia de ligação $3 \mathrm{~d}_{5 / 2}$ corresponde ao valor de paládio no estado metálico ${ }^{30}$. Na presença de cloreto, o paládio não pode ser completamente reduzido, assim a energia de ligação $\mathrm{Pd} 3 \mathrm{~d}_{5 / 2}$ é maior. Entretanto, na amostra de $\mathrm{Pd} / \mathrm{MoO}_{3} / \mathrm{Al}_{2} \mathrm{O}_{3}$ submetida a tratamento HTR, o metal encontra-se em um estado mais reduzido, logo é mais fácil extrair o elétron do seu subnível 3d. O espectro de XPS do $\mathrm{Pd} / \mathrm{Al}_{2} \mathrm{O}_{3}$ sem cloreto, submetido a tratamento HTR mostrou uma resolução muito ruim, sendo que sua energia de ligação $\operatorname{Pd} 3 \mathrm{~d}_{5 / 2}$ não pôde ser calculada com um desvio satisfatório,

Tabela 1. Energia de ligação obtida por XPS

\begin{tabular}{lccc}
\hline Amostra & $\begin{array}{c}\text { Tratamento } \\
\text { térmico }\end{array}$ & \begin{tabular}{c}
${\mathrm{Pd} 3 \mathrm{~d}_{5 / 2}}_{(\mathrm{eV})^{\mathrm{a}}}$ \\
\hline $\mathrm{Pd} / \mathrm{Al}_{2} \mathrm{O}_{3}$ com cloreto
\end{tabular} & $\begin{array}{c}\mathrm{Mo} \mathrm{3d}_{5 / 2} \\
(\mathrm{eV})^{\mathrm{a}}\end{array}$ \\
${\mathrm{Pd} / \mathrm{Al}_{2} \mathrm{O}_{3} \text { sem cloreto }}_{\mathrm{Pd} / \mathrm{MoO}_{3} / \mathrm{Al}_{2} \mathrm{O}_{3}}^{\text {LTR }}$ & 336,4 & \\
Paládio metálico & $\mathrm{HTR}$ & 335,5 & \\
Óxido de molibdênio (VI) & & 334,7 & 232,0 \\
\hline
\end{tabular}

${ }^{\mathrm{a}}=$ desvio de $0,2 \mathrm{eV}$;

${ }^{\mathrm{b}}=$ obtido da referência 35 ;

${ }^{\mathrm{c}}=$ obtido da referência 36 
possivelmente devido a forte interação do paládio com o oxigênio da alumina. Essa interpretação é também suportada pela razão $\mathrm{Pd} / \mathrm{Al}$ que será discutida abaixo. A energia de ligação $\mathrm{Mo} 3 \mathrm{~d}_{5 / 2}$ do $\mathrm{Pd} / \mathrm{MoO}_{3} /$ $\mathrm{Al}_{2} \mathrm{O}_{3}$ é também apresentada na Tabela 1, sendo possível observar um deslocamento para menores valores quando comparado com a energia de ligação Mo $3 \mathrm{~d}_{5 / 2}$ do óxido de molibdênio (VI) ${ }^{31}$. Esse resultado é uma evidência adicional da presença do efeito SMSI, que é devido a redução parcial do molibdênio do suporte.

A razão atômica $\mathrm{Pd} / \mathrm{Al}$, obtida na análise de XPS é mostrada na Tabela 2. A razão $\mathrm{Pd} / \mathrm{Al}$ foi quase constante para os catalisadores, exceto para a amostra de $\mathrm{Pd} / \mathrm{Al}_{2} \mathrm{O}_{3}$ submetido ao tratamento HTR. A profundidade de análise da técnica XPS é de $c a .2 \mathrm{~nm}$, portanto o decréscimo na razão $\mathrm{Pd} / \mathrm{Al}$, observado nessa amostra, com relação aquela submetida ao tratamento LTR, foi interpretado considerando-se a migração do metal da superfície para o bulk (interior da amostra), devido a interação do metal com a alumina.

Tabela 2. Razão atômica Pd/Al obtida por XPS

\begin{tabular}{lcc}
\hline Amostras & $\begin{array}{c}\text { Tratamento } \\
\text { térmico }\end{array}$ & $\begin{array}{c}\text { Razão atômica } \\
\mathrm{Pd} / \mathrm{Al}\end{array}$ \\
\hline $\mathrm{Pd} / \mathrm{Al}_{2} \mathrm{O}_{3}$ sem cloreto & LTR & 0,04 \\
& HTR & 0,02 \\
$\mathrm{Pd} / \mathrm{Al}_{2} \mathrm{O}_{3}$ com cloreto & LTR & 0,05 \\
$\mathrm{Pd} / \mathrm{MoO}_{3} / \mathrm{Al}_{2} \mathrm{O}_{3}$ & HTR & 0,04 \\
\hline
\end{tabular}

Nesse trabalho nós consideramos que o envenenamento por cloreto é um efeito eletrônico e é o responsável pelo deslocamento das bandas de carbonila coordenada nos espectros da Figura 2 em relação aos espectros da Figura $1\left(+22 \mathrm{~cm}^{-1}\right)$. Entretanto, é possível produzir um efeito eletrônico contrário ao do envenenamento por cloreto, desde que seja usado um suporte com propriedades redutíveis. Portanto este trabalho sugere que o uso de suportes redutíveis pode produzir uma inibição parcial do envenenamento por cloreto. Catalisadores com suportes mistos, onde um dos óxidos é redutível, foram muito estudados nos últimos anos ${ }^{32-36}$, sendo que esses sistemas mostram melhor atividade catalítica que os catalisadores que usam alumina pura como suporte ${ }^{32-34}$.

\section{CONCLUSÕES}

No catalisador $\mathrm{Pd} / \mathrm{Al}_{2} \mathrm{O}_{3}$ com cloreto o paládio não pode ser totalmente reduzido, possivelmente devido a formação de espécies $\mathrm{Pd}$ $\mathrm{Cl}$, portanto o envenenamento por cloreto é atribuído a efeito de natureza eletrônica.

No catalisador $\mathrm{Pd} / \mathrm{Al}_{2} \mathrm{O}_{3}$ sem cloreto, submetido a tratamento LTR, o paládio está em um estado mais reduzido que nas amostras que apresentam cloreto. Portanto uma forte ligação pi (retro-doação) entre o paládio e a carbonila é formada. Entretanto, o tratamento HTR pode produzir um efeito similar ao envenenamento por cloreto devido a interação do metal com o oxigênio da superfície do supor- te. O efeito SMSI pode ser usado para aumentar a densidade eletrônica do metal disperso resultando em um efeito contrário ao envenenamento por cloreto.

\section{AGRADECIMENTOS}

À Fapergs pelo financiamento e bolsa IC para E. V. Cunha e ao PIBIC-CNPq pela bolsa IC para F. Faccin.

\section{REFERÊNCIAS}

1. Manoli, J.-M.; Potvin, C.; Muhler, M.; Wild, U.; Resofszki, G.; Buchholz, T.; Paál, Z.; J. Catal. 1998, 178, 338.

2. Waghray, A.; Wang, J.; Oukaci, R.; Blackmond, D. G.; J. Phys. Chem. 1992, 96, 5954.

3. Gloor, A. P.; Prins, R.; Recl. Trav. Chim. Pays-Bas. 1994, 113, 481

4. Almusaiteer, K.; Chuang, S. C.; J. Catal. 1999, 184, 189.

5. Tavoularis, G.; Keane, M. A.; Appl. Catal., A 1999, 182, 309.

6. Tavoularis, G.; Keanem, M. A.; J. Mol. Catal. A: Chem. 1998, 142, 187.

7. Bastein, A. G. T. M.; van der Boogert, W. J.; van der Lee, G.; Luo, H.; Schuller, B.; Ponec, V.; Appl. Catal. 1987, 29, 243.

8. Gloor, A. P.; Prins, R.; J. Phys. Chem. 1994, 98, 9865.

9. Hicks, R. F.; Qi, H.; Young, M. L.; Lee, R. G.; J. Catal. 1990, 122, 295.

10. Cullis, C. F.; Willatt, B. M.; J. Catal. 1984, 86, 187.

11. Klier, K.; Simmons, G. W.; Park, K. T.; Wang, Y.-N.; Hess, J. S.; Herman, R. G.; Langmuir 1998, 14, 1384.

12. Simone, D. O.; Kennelly, T.; Brungard, N. L.; Farrauto, R. J.; Appl. Catal. 1991, 70, 87.

13. Marécot, P.; Fakche, A.; Kellali, B.; Mabilon, G.; Prigent, M.; Barbier, J.; Appl. Catal. B: Envirom. 1994, 3, 283.

14. Spencer, M. S.; Top. Catal. 1999, 8, 259.

15. Barbier, J.; Bahlou, D.; Marécot, P.; Catal. Lett. 1991, 8, 327.

16. Foschiera, J. L.; Pizzolato, T. M.; Benvenutti, E. V.; J. Braz. Chem. Soc. 2001, 12, 159

17. Scofield, J. H.; J. Electron Spectrosc. Relat. Phenom. 1976, 8, 129.

18. Hoost, T. E.; Otto, K.; Laframboise, K. A.; J. Catal. 1995, 155, 303.

19. Aylor, A. W.; Lobree, L. J.; Reimer, J. A.; Bell, A. T.; J. Catal. 1997, 172, 453.

20. Santiesteban, J. G.; Calabro, D. C.; Borghard, W. S.; Chang, C. D.; Vartuli, J. C.; Tsao, Y. P.; Natal-Santiago, M. A.; Bastian, R. D.; J. Catal. 1999, $183,314$.

21. Dandekar, A.; Vannice, M. A.; J. Catal. 1999, 183, 344.

22. Arai, M.; Obata, A.; Nishiyama, Y.; J. Catal. 1997, 166, 115.

23. Resasco, D. E.; Weber, R. S.; Sakellson, S.; McMillan, M.; Haller, G. L.; J. Phys. Chem. 1988, 92, 189.

24. Benvenutti, E. V.; Franken, L.; Moro, C. C.; Davanzo, C. U.; Langmuir 1999, 15, 8140.

25. Yoshitake, H.; Iwasawa, Y.; J. Phys. Chem. 1992, 96, 1329.

26. Benvenutti, E. V.; Davanzo, C. U.; Quim. Nova, 1999, 22, 674.

27. Englisch, M.; Jentys, A.; Lercher, J. A.; J. Catal. 1997, 166, 25.

28. Ishihara, T.; Harada, K.; Egushi, K.; Arai, H.; J. Catal. 1992, 136, 161.

29. Vannice, M. A.; J. Mol. Catal. 1990, 59, 165

30. Peuckert, M.; J. Phys. Chem. 1985, 89, 2481

31. Fleisch, T. H.; Mains, G. J.; J. Chem. Phys. 1982, 76, 780.

32. Farrauto, R. J.; Voss, K. E.; Appl. Catal., B 1996, 10, 29.

33. Farrauto, R. J.; M.Heck, R.; Catal. Today 1999, 51, 351.

34. Oh, S. H.; Eickel, C. C.; J. Catal. 1993, 112, 543.

35. Marécot, P.; Pirault, L.; Mabilon, G.; Prigent, M.; Barbier, J.; Appl. Catal., $B$ 1994, 5, 57

36. Shyu, J. Z.; Otto, K.; Watkins, W. L. H.; Graham, G. W.; Belitz, R. K.; Gandhi, H. S.; J. Catal. 1988, 114, 23. 